\title{
ALGORITHMIC COMPLEXITY OF TERM REWRITING SYSTEMS
}

\author{
C. CHOPPY, S. KAPLAN, M. SORIA \\ Laboratoire de Recherche en Informatique \\ U.A. C.N.R.S. 410 \\ Université Paris-Sud, Bât 490 \\ F-91405 ORSAY Cédex, FRANCE \\ net : mcvax!inria!lri!cc, mcvax!inria!lri!kaplan, mcvax!inria!lri!soria
}

\section{Introduction}

Algebraic specifications are now widely used for data structuring and they tum out to be quite useful for various aspects of program development, such as prototyping, assisted program construction, proving properties, etc [BCV 85, FG 84, FGJM 84, GHW 85, Kap 86]. Some of these applications require to add a notion of computation to algebraic specifications, for instance by providing a (convergent) rewrite rule system that expresses the properties of the operators. In this context, it may be of first interest to define a notion of algorithmic complexity for an algebraic specification, or, more precisely, a notion of complexity for each operator defined in the specification. Computing operator complexity within a given specification helps understanding how evaluation costs are distributed; it may point out "costly" operators, and motivate the search for an equivalent, but "cheaper", specification.

In [CLR 80], the cost of a term is defined as the number of rewriting steps for reducing it to its normal form, and the cost of an operator is defined as the general cost of a term obtained by applying this operator to terms in normal form. In this paper, we further formalize this notion of operator complexity and investigate its computation through analysis methods developed for instance in [Stey 84] and [Fla 87]. We show how these methods apply to the computation of the enumerative series over the terms of an algebraic specification. We define the notion of regular rewriting systems, and consider cost series of operators that are described by such systems. We show how these analysis methods apply to compute such costs and provide an asymptotic evaluation of the average cost of an operator. Our results allow costs to be computed without any explicit manipulation of series. We provide an eventual user with ready-to-use formulae, where the different parameters only depend on the "geometry" of the system, e.g. the number of constructors in the left handside of rules, number of occurrences of a derived operator in the right handside, etc.

Quantitative evaluation of rewriting systems had not yet been studied under such an approach (except in [CLR 80]), to our knowledge. From a different point of view, complexity of algebraic implementations has been studied in [BBWT 81, E\&M 81, etc.] w.r.t. computability issues.

\section{Introductory example}

Let us assume that one wants to evaluate the average cost of a given computation on some data set. The data belong to a set of objects, a size can be computed for each object; let $D_{n}$ denote the set of 
objects of size $n$, and $N_{n}=$ card $\left(D_{n}\right)$. Assuming all the objects have the same probability, the average cost is [GSF 86] :

$$
\overline{\mathrm{C}}_{\mathrm{n}}=\frac{1}{\mathrm{~N}_{\mathrm{n}}} \sum_{\mathrm{d} \in \mathrm{D}_{\mathrm{a}}} \operatorname{cost}(\mathrm{d})=\frac{\mathrm{C}_{\mathrm{n}}}{\mathrm{N}_{\mathrm{n}}} .
$$

Generating series are defined by associating the series $a(z)=\sum a_{n} z^{n}$ to a sequence $\left(a_{n}\right)$ : to the sequence $\left(N_{n}\right)$ is associated the enumerative series $N(z)=\sum N_{n} z^{n}$, and to the sequence $\left(C_{n}\right)$ is associated the cost series $C(z)=\sum C_{n} z^{n}$. Computation of the coefficients of the generating series can be performed either using "exact" methods (e.g. using the Lagrange inversion theorem) or methods that provide an approximation, based on real or complex analysis techniques. The asymptotic value of the coefficients $a_{n}$ of a complex series $\sum a_{n} z^{n}$ may be evaluated using results of complex functions theory (essentially based on Cauchy formula) : the singularity closest to the origin determines the order of growth of the coefficients (more precisely, their exponential factor is determined by convergence radius of the series and their polynomial factor is function of the nature of the singularity) [Fla 87].

Consider the example of a specification of binary trees with two constructors : the constant ' $a$ ' and the '..' operator (think of "cons" in Lisp).

Considering the enumerative series : $N_{t r e e}(z)=\sum_{n \geq 0} N_{n} z^{n}$, since each nth power of $z$ appears as many times as there are trees of size $n$, we have : $N_{\text {tree }}(z)=\sum_{t \in T_{\text {tre }}} z^{|t|}$ where $T_{\text {tree }}$ is the set of terms built with the constructors 'a' and '...'.

Let us first perform the computation of $\mathrm{N}_{\text {tree }}(z)$ by case analysis on terms :

$$
N_{\text {tree }}(z)=\sum_{t=a} z^{|t|}+\sum_{t=t_{1} \cdot t_{2}} z^{\left|t_{1} \cdot t_{2}\right|}
$$

Since $\left|t_{1} \cdot t_{2}\right|=1+\left|t_{1}\right|+\left|t_{2}\right|: \quad N_{\text {tree }}(z)=z+z \sum_{t_{1} \in T_{\text {troe }}} \sum_{t_{n} \in T_{\text {tree }}} z^{\left|t_{1}\right|} z^{\left|t_{2}\right|}=z\left(1+N_{\text {tree }}^{2}(z)\right)$.

In the general case, computation of $N_{\text {tree }}(z)$ can also be performed using systematic methods for computing enumerative series and cost series for algorithms on combinatorial structures [Fla 87] (in particular, one may apply these methods when trees are used as data structures to represent terms); the main steps of these methods are the following :

- take the construction primitives of the combinatorial object and deduce the structural equations ; in the case of tree this leads to:

$$
\text { tree }=a+i_{\text {tree }} \text { tree } \text { or } \text { tree }=a+. \times \text { tree } \times \text { tree }
$$

where + is the disjoint union and $x$ the cartesian product

- transpose the structural equation(s) to generating series, using the fact that, when considering the associated series, any disjoint union is expressed by a sum and any cartesian product is expressed by a product ; in our example, this leads to :

$$
N_{\text {tree }}(z)=z\left(1+N_{\text {tree }}^{2}(z)\right)
$$


(the same result was obtained above by case analysis)

- solve the generating series equarions. In the above example, simple resolution leads to : $N_{\text {tree }}(z)=\frac{1-\sqrt{1-4 z^{2}}}{2 z}$ (this solution is adequate since it is analytic at the origin), and further computation by series development leads $N_{2 p}=0$ and to $N_{2 p+1}=\frac{1}{p+1}\left[\begin{array}{l}2 p \\ p\end{array}\right]$ (Catalan numbers, cf. [Knu 73]). Using the Stirling formula: $p i=\sqrt{2 \pi p}\left[\frac{p}{e}\right]^{p}\left(1+o\left(\frac{1}{p}\right)\right)$, this yields :

$$
\mathrm{N}_{2 \mathrm{p}+1}=\frac{1}{\sqrt{\pi}} \mathrm{p}^{-3 / 2} 2^{2 \mathrm{p}}\left(\mathrm{I}+\mathrm{O}\left(\frac{1}{\mathrm{p}}\right)\right)
$$

Another approach is to use complex analysis methods (local analysis around singularities) for passing from functional equations over generating functions to asymptotic expressions of their coefficients. Continuing with our example, we have : $N_{\text {tree }}(z)=-\frac{\sqrt{1-2 z} \sqrt{1+2 z}}{2 z}+\frac{1}{2 z}$

$N_{\text {tree }}(z)$ is analytic for $|z|<\frac{1}{2}$ and has two singularities : $z=\frac{1}{2}$ and $z=-\frac{1}{2}$.

Let us apply the Newton expansion : $\left[z^{n}\right](1-k z)^{\alpha \dagger}=k^{n}(-1)^{n}\left[\begin{array}{l}n \\ \alpha\end{array}\right]=k^{n}\left[\begin{array}{c}n-\alpha-1 \\ -\alpha-1\end{array}\right]$

One shows [Fla 87] that when $n \rightarrow+\infty:\left[z^{n}\right](1-k z)^{\alpha}=\frac{n^{-\alpha-1}}{\Gamma(-\alpha)} k^{n}\left(1+O\left(\frac{1}{n}\right)\right)$

(where $\Gamma$ is the Euler Gamma function: $\Gamma(x)=\int_{0}^{+\infty} e^{-t} t^{x-1} d t$. We recall that $\Gamma(x+1)=x \Gamma(x)$, $\Gamma(1)=1$ - hence $\Gamma(n)=(n-1) !$ when $n \in N$ - and $\left.\Gamma\left(\frac{1}{2}\right)=\sqrt{\pi}\right)$.

The contributions of singularities with same module are added together, leading in our case to:

$\left[z^{n}\right] N(z)=\frac{1}{\sqrt{2 \pi}} 2^{n+1} n^{-3 / 2}\left(1+O\left(\frac{1}{n}\right)\right)$ for $n$ odd, and 0 for $n$ even.

A more systematic approach that uses "transfer lemmas" [Fla 87] is presented in section 2.

Now let us add a derived operator " $\uparrow$ _ (called "shuffe" since it is moving subtrees around) defined with the following rules:

$$
\left(S_{1}\right): a \uparrow t \rightarrow a \cdot t, \quad\left(t_{1} \cdot t_{2}\right) \uparrow a \rightarrow\left(t_{1} \cdot t_{2}\right) \cdot a, \quad\left(t_{1} \cdot t_{2}\right) \uparrow\left(u_{1}, u_{2}\right) \rightarrow\left(t_{1} \uparrow u_{1}\right) \cdot\left(t_{2} \uparrow u_{2}\right)
$$

The cost of a term is the number of rewriting steps necessary to reduce it to its normal form for a given strategy ; the cost of an operator is defined as the cost of a term obtained by applying this operator to the terms in normal form. In all examples considered in this paper, it is insured that operator costs are independent from the evaluation strategy (cf. propsition in section 3).

Let us evaluate the cost functions for the operators of this specification : ' $a$ ' and '_. ' ' being constructors, the corresponding cost functions are equal to zero. Computation of the cost function for the operation "_ $\uparrow$ _' will be done by means of the following generating function (cf. definition 3.4):

$C^{\uparrow}(z)=\sum_{n \geq 0} C_{n}^{\uparrow} z^{n} \quad$ where $C_{n}^{\uparrow}=\sum_{\substack{t, u \in T_{\text {wow }} \\|t|+|u|=n}} \operatorname{cost}(t \uparrow u) \quad$ (where $|t|$ is the size of the term $t$, i.e. the

$f^{n}\left[z^{n}\right](z)$ denotes the coefficient of $z^{n}$ in $\Phi(z)$ 
total number of symbols that appear in $t$, and where $T_{\text {tree }}$ is the set of terms in normal form, which is exactly here the set of terms built on the constructors ' $a$ ' and '. . .').

Hence : $C^{\top}(z)=\sum_{t, u \in T_{\text {tro }}} \operatorname{cost}(t \uparrow u) z^{|t|+|u|}$

Let us use a case analysis for terms in normal form to compute $\mathrm{C}^{\uparrow}(\mathrm{z})$ :

$$
\begin{aligned}
C^{\uparrow}(z)= & \sum_{t \in T_{\text {cote }}} \operatorname{cost}(a \uparrow t) z^{1+|t|}+\sum_{t_{1}, t_{2} \in T_{\text {wos }}} \operatorname{cost}\left(\left(t_{1} \cdot t_{2}\right) \uparrow a\right) z^{\left|t_{1} \cdot t_{2}\right|+1} \\
& +\sum_{t_{1}, t_{2}, u_{1}, u_{2} \in T_{\text {troe }}} \operatorname{cost}\left(\left(t_{1} \cdot t_{2}\right) \uparrow\left(u_{1} \cdot u_{2}\right)\right) z^{\left|t_{1}, t_{2}\right|+\left|u_{1}, u_{2}\right|}
\end{aligned}
$$

since : $\quad \operatorname{cost}(a \uparrow t)=1+\cos t(a \cdot t)=1$,

$$
\begin{aligned}
& \operatorname{cost}\left(\left(t_{1} \cdot t_{2}\right) \uparrow a\right)=1+\operatorname{cost}\left(\left(t_{1} \cdot t_{2}\right) \cdot a\right)=1, \\
& \operatorname{cost}\left(\left(t_{1}, t_{2}\right) \uparrow\left(u_{1} \cdot u_{2}\right)\right)=1+\operatorname{cost}\left(\left(t_{1} \uparrow u_{1}\right) \cdot\left(t_{2} \uparrow u_{2}\right)\right) \\
& =1+\operatorname{cost}\left(t_{1} \uparrow u_{1}\right)+\operatorname{cost}\left(t_{2} \uparrow u_{2}\right),
\end{aligned}
$$

we have :

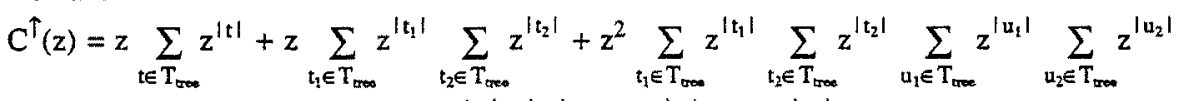

$$
\begin{aligned}
& +z^{2} \sum_{t_{1}, u_{1} \in T_{\text {cose }}} \operatorname{cost}\left(t_{1} \uparrow u_{1}\right) z^{\left|t_{1}\right|} z^{\left|u_{1}\right|} \sum_{t_{2} \in T_{t+\infty}} z^{\left|t_{2}\right|} \sum_{u_{2} \in T_{\text {trox }}} z^{\left|u_{2}\right|} \\
& +z^{2} \sum_{t_{2}, u_{2} \in T_{00}} \operatorname{cost}\left(t_{2} \uparrow u_{2}\right) z^{\left|t_{2}\right|} z^{\left|u_{2}\right|} \sum_{t_{1} \in T_{0 \infty}} z^{\left|t_{1}\right|} \sum_{u_{3} \in T_{\text {pos }}} z^{\left|u_{1}\right|} \\
& =z N_{\text {tree }}(z)+z^{2} N_{\text {tree }}^{2}(z)+z^{2} N_{\text {tree }}^{4}(z)+2 z^{2} N_{\text {tree }}^{2}(z) C^{\uparrow}(z) \text {. }
\end{aligned}
$$

Hence :

$$
C^{\dagger}(z)=\frac{z N_{\text {tree }}(z)+z^{2} N_{\text {tree }}^{2}(z)+z^{2} N_{\text {tree }}^{4}(z)}{1-2 z^{2} N_{\text {tree }}^{2}(z)}=\frac{z N_{\text {tree }}(z)+z N_{\text {tree }}^{3}(z)}{1-2 z^{2} N_{\text {tree }}^{2}(z)}=\frac{N_{\text {tree }}^{2}(z)}{1-2 z^{2} N_{\text {tree }}^{2}(z)}
$$

Replacing in this expression $N_{t r e e}(z)$ by its value : $\frac{1-\sqrt{1-4 z^{2}}}{2 z}$ and using the same complex analysis method as for $\mathrm{N}_{\text {tree }}(\mathrm{z})$ (development around singularities, Newton expansion, adding up the contributions of singularities) yields to : $C_{2 p}^{\uparrow}=\frac{4}{\sqrt{\pi}} 2^{2 p} p^{-3 / 2}\left(1+O\left(\frac{1}{p}\right)\right)$

Now the average cost is : $\bar{C}_{2 p}^{\uparrow}=\frac{C_{2 p}^{\uparrow}}{N_{2,2 p}}$ where $N_{2,2 p}$ (cf. section 2) is the number of tree couples $\left(t_{1}, t_{2}\right.$ ) such that $\left|t_{1}\right|+\left|t_{2}\right|=2 p$. Computation of $N_{2,2 p}$ yields :

in this case, the average cost is constant (asymptotically).

$$
C_{2 p}^{\uparrow}=4\left(1+O\left(\frac{1}{p}\right)\right)
$$

We develop in section 3, a general method for computing the coefficients of series such as $C^{\uparrow}(z)$, when the rules defining " $\uparrow$, constitute a regular rewriting system.

\footnotetext{
${ }^{\dagger \dagger}$ using the fact that $: N_{\text {tree }}(z)=z\left(1+N_{\text {tree }}^{2}(z)\right)$
} 


\section{Enumerative series of the term algebra}

We denote by $T_{\text {Constr }}$ the set of terms built on the signature Constr. Let us denote by $\alpha_{k}$ the number of symbols in Constr of arity $k$. We always suppose that $\alpha_{0} \neq 0$.

\section{Definition 2.1}

Let $N_{n}$ stand for the number of terms in $T_{\text {Constr }}$ of size $n$. The enumerative series of $T_{\text {Constr }}$ is :

$$
N(z)=\sum_{n \geq 0} N_{n} z^{n}=\sum_{t \in T_{\cos x}} z^{|n|}
$$

Let $\Phi(X)$ stand for the polynomial : $\Phi(X)=\sum_{k=0}^{p} \alpha_{k} X^{k}$, where ' $p$ ' denotes the largest arity in Constr, and where there exists $\alpha_{k}>0$ with $k \geq 2 .^{\dagger}$

We have the following resuits :

(1) $N(z)$ is a solution of the functional equation: $N(z)=z . \Phi(N(z)$ ) (cf. section 1 : transposition from structural equations to generating series).

(2) Let us suppose that there is no polynomial $\Psi^{\prime}$ and integer $d \geq 2$ such that $\Phi(X)=\Psi\left(X^{d}\right)$; let $\tau$ be the smallest root of the equation $\Phi(X)=X \Phi^{\prime}(X)$, and $p=\frac{\tau}{\Phi(\tau)} ; p$ is the convergence radius of $N(z)$ and $0<\rho<1$. $\tau$ is the only real positive root of the equation $\Phi(X)=X \Phi^{\prime}(X)$ such that $[X]=\tau$. Moreover, $\tau=N(\rho)$ and $\rho$ is the only singularity of $N(z)$ such that $|z|=\rho$. Then around $z=\rho$ [M\&M 78] :

$$
N(z)=\tau-\sqrt{\frac{2 \Phi(\tau)}{\Phi^{\prime \prime}(\tau)}}\left[1-\frac{z}{\rho}\right)^{1 / 2}+\gamma\left(1-\frac{z}{\rho}\right)+O\left(\left[1-\frac{z}{\rho}\right)^{3 / 2}\right)
$$

(3) Transfer Lemmas : when it is possible to have an asymptotic development of a series around the singularity that is closest to the origin, under some conditions (that are always fulfilled in the case of term algebras) an estimation of the series coefficients can be deduced from the series estimation through transfer lemmas [Fla 87].

We use the transfer lemmas in the following case :

Let $f(z)=h(z)+O(g(z))$ be the expansion of $f$ around the singularity $p$, where $f$ and $g$ are standard functions of the type $\left(1-\frac{z}{\rho}\right)^{\alpha}$ and $h$ is of higher order than $g$ around $\rho$. Then : $\left[z^{n}\right] f(z)=\left[z^{n}\right] h(z)+O\left(\left[z^{n}\right] g(z)\right)$.

Considering the expression (F1) and applying the transfer lemmas, one gets :

$$
N_{n}=-\sqrt{\frac{2 \Phi(\tau)}{\Phi^{\prime \prime}(\tau)}} \frac{\rho^{-n} n^{-3 / 2}}{\Gamma(-1 / 2)}+0\left[\rho^{-n} n^{-5 / 2}\right] \quad \text { with } \Gamma(-1 / 2)=-2 \sqrt{\pi}
$$

i.e. finally :

$$
\mathrm{N}_{\mathrm{n}}=\sqrt{\frac{\Phi(\tau)}{2 \pi \Phi^{\prime \prime}(\tau)}} \rho^{-\mathrm{n}} \mathrm{n}^{-3 / 2}\left(1+O\left(\frac{1}{\mathrm{n}}\right)\right)
$$

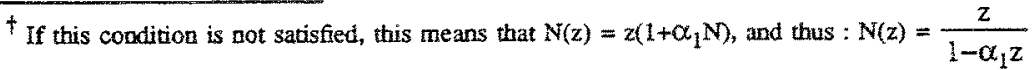


Note 1 :

For average cost computations, we need to evaluate the quantity $\mathrm{N}_{\mathrm{m}, \mathrm{n}}$, that is the number of terms $t_{1}, \ldots, t_{m} \in T_{\text {Constr }}$ such that $\left|t_{1}\right|+\ldots+\left|t_{m}\right|=n$. Then :

$$
\sum_{n=0}^{\infty} N_{m, n} z^{n}=\sum_{n=0}^{\infty} \sum_{\substack{t_{1}, \ldots, t_{m} \in T_{\text {Conar }} \\\left|t_{1}\right|+\ldots+t_{n} \mid=n}} z^{n}=\sum_{t_{1}, \ldots, t_{m} \in T_{\text {Coum }}} z^{\left|t_{1}\right|+\ldots+\left|t_{m}\right|}=(N(z))^{m} .
$$

Thus, $\mathrm{N}_{\mathrm{m}, \mathrm{n}}$ is the coefficient of $\mathrm{z}^{\mathrm{n}}$ in $(\mathrm{N}(\mathrm{z}))^{\mathrm{m}}$, and using (F1) we have :

$(\mathrm{N}(\mathrm{z}))^{\mathrm{m}}=\tau^{\mathrm{m}}+\mathrm{m} \tau^{\mathrm{m}-1} \sqrt{\frac{2 \Phi(\tau)}{\Phi^{\prime \prime}(\tau)}}\left[1-\frac{z}{\rho}\right]^{1 / 2}+\gamma 2\left[1-\frac{\mathrm{z}}{\rho}\right]+\mathrm{O}\left[\left(1-\frac{z}{\rho}\right]^{3 / 2}\right]$.

Now, using the transfer lemmas, we obtain :

$$
N_{m, n}=m \tau^{m-1} \sqrt{\frac{\Phi(\tau)}{2 \pi \Phi^{\prime \prime}(\tau)}} \rho^{-n} n^{-3 / 2}\left(1+O\left(\frac{1}{n}\right)\right)
$$

and : $N_{m, n}=m \tau^{m-1} N_{n}=\left.\frac{\partial}{\partial N}\left(N^{m}(z)\right)\right|_{z=\rho} N_{n}$

More generally, given the polynomial on $N(z): P(z)=\sum_{i=1}^{q} \alpha_{i} N^{i}(z)$ similarly $:\left[z^{n}\right] P(z)=\left.N_{n} \frac{\partial P}{\partial N}\right|_{z=0}$.

\section{Note 2 :}

The above results were obtained for the case where $\rho$ was the only singularity of $N(z)$ such that $|z|=\rho$. Let us now consider the case where there are more than one such singularity. If $\Phi(X)=\Psi\left(X^{d}\right)$, let $\tau$ still denote the unique real, positive root of the equation $\Phi(X)=X \Phi^{\prime}(X)$. The other solutions of this equation are $e^{2 i k \pi d d} \tau$, for $k=1,2, \ldots, d-1$. We now have :

$$
\begin{aligned}
& N_{n}=d \sqrt{\frac{\Phi(\tau)}{2 \pi \Phi^{\prime \prime}(\tau)}} \rho^{-n} n^{-3 / 2}\left(1+O\left(\frac{1}{n}\right)\right) \quad \text { if } n \equiv 1[\bmod d] \\
& N_{n}=0 \text { otherwise. }
\end{aligned}
$$

and :

$$
\begin{aligned}
& N_{m, n}=d m \tau^{m-1} \sqrt{\frac{\Phi(\tau)}{2 \pi \Phi^{\prime \prime}(\tau)}} \rho^{-n} n^{-3 / 2}\left(1+O\left(\frac{1}{n}\right)\right) \text { if } n \equiv m[\bmod d] \\
& N_{m, n}=0 \text { otherwise. }
\end{aligned}
$$

\section{Example :}

These results immediately apply to the example of section 1 where Constr $=\left\{a_{-}-{ }_{-}\right\}$. We have $\alpha_{0}=1, \alpha_{1}=0, \alpha_{2}=1$. Then $\Phi(X)=1+X^{2}$, of the form $\Psi\left(X^{2}\right)$, with $\Psi(Y)=1+Y$. $\tau$ satisfies : $1+\tau^{2}=2 \tau^{2}$. Thus $\tau=1$ and $\rho=\frac{\tau}{\Phi(\tau)}=1 / 2$. , and we derive :

$$
\begin{aligned}
& N_{n}=\frac{1}{\sqrt{2 \pi}} 2^{n+1} n^{-3 / 2}\left(1+O\left(\frac{1}{n}\right)\right) \text { for } n \text { odd, and } 0 \text { for } n \text { even } \\
& N_{m, n}=\frac{m}{\sqrt{2 \pi}} 2^{n+1} n^{-3 / 2}\left(1+O\left(\frac{1}{n}\right)\right) \text { for } n \equiv m[\bmod d] \text {, and } 0 \text { otherwise. }
\end{aligned}
$$

Notice that letting $m=2, n=2 p$, we obtain : $N_{2,2 p}=\frac{1}{\sqrt{2 \pi}} 2^{2 p} p^{-3 / 2}\left(1+O\left(\frac{1}{p}\right)\right)$ which leads to the same result as obtained in section 1. 


\section{Cost series for the derived operators}

We suppose that the set of operator symbols $\Sigma$ is partitioned into:

- a set Constr of constructors (these are functions that generate the set of terms, and for which the generating function $N$ was computed hereabove);

- a set Der of derived operators (that realize computations on terms of $\mathrm{T}_{\text {Constr }}$ ).

We wish to ensure that any term of $\mathrm{T}_{\text {Constr }}$ (i.e. built with constructors only) is irreducible, and that for $f \in$ Der and for $t_{1}, \ldots, t_{n} \in T_{\text {Constr }}, f\left(t_{1}, \ldots, t_{n}\right)$ rewrites into a unique term of $T_{\text {Constr }}$ in a finite amount of rewrite steps. To this effect, we are going to restrict the form of the rules that are acceptable.

\section{Definition 3.1}

A Constr-enumeration associated to an operator $f \in D e r$, with $\operatorname{ar}(\mathrm{f})=\mathrm{n}$, is a finite family of $\mathrm{n}$-tuples $\left(\vec{\omega}_{e}\right)_{1 \leq e \leq D(f)}$ of $\left(T_{\text {Constr }}(X)\right)^{\mathrm{n}}$ such that :

- each $\bar{\alpha}_{\mathrm{e}}$ contains at least a constructor symbol

- for any $\vec{t} \in\left(T_{\text {Constr }}\right)^{\mathrm{n}}$, there exists a unique substitution $\sigma: X \rightarrow \mathrm{T}_{\text {Constr }}$ and a unique e such that: $\quad \vec{t}=\vec{\omega}_{\mathrm{e}} \sigma$

Thus, intuitively speaking, the $\left(\omega_{e}\right)$ form a description of the possible arguments of $f$, that is at the same time exhaustive and non-ambiguous. For instance, with Constr $=\{0, s\}$ and $\operatorname{Der}=\{+\}$, we can take : $\vec{w}_{1}=(x, 0)$ and $\vec{w}_{2}=(x, s(y))$, as a Constr-enumeration of the symbol ' $t$ '.

Let $\mathrm{X}_{\mathrm{e}}$ stand for the variables that appear in $\vec{\omega}_{e}$, and $\xi_{e}$ for the number of constructors that appear in $\vec{\omega}_{e}$ (let us recall that, according to Definition $3,1, \xi_{e} \neq 0$ ). In the previous example, we have : $X_{1}=\{x\}, \xi_{1}=i$ and $X_{2}=\{x, y\}, \xi_{2}=1$.

\section{Definition 3.2}

A Constr-definition of $f \in$ Der is a set of rewrite rules $R_{\mathrm{f}}=\left(\mathrm{r}_{\mathrm{e}}\right)_{1 \leq e \leq \mathrm{D}(\mathrm{f})}$ such that :

- each rule is of the form $r_{e}: f\left(\omega_{e}\right) \rightarrow \rho_{e}$, where $\left(\vec{\omega}_{e}\right)_{e \in D(f)}$ is a Constr-enumeration of $f$;

- each $\rho_{\mathrm{e}}$ is of the form $\rho_{\mathrm{e}}=\mathrm{K}\left(\mathrm{x}_{1}, \ldots, \mathrm{x}_{\mathrm{n}}, \phi_{1}, \ldots, \phi_{\mathrm{m}}\right)$, where :

- $\mathrm{K}$ is a context made of constructors only,

- $\left|\mathrm{x}_{1}, \ldots, \mathrm{x}_{\mathrm{n}}\right| \subseteq \mathrm{X}_{\mathrm{e}}$,

- each $\phi_{\mathrm{x}}$ is of the form $g\left(y_{1}, \ldots, y_{\text {ar }(g)}\right)$, where $g$ is a derived operator, $\left\{y_{1}, \ldots, y_{\operatorname{ar}(g)}\right\} \subseteq x_{e}$, and $y_{i} \neq y_{j}$ if $i \neq j$. 
A typical rule thus looks like :

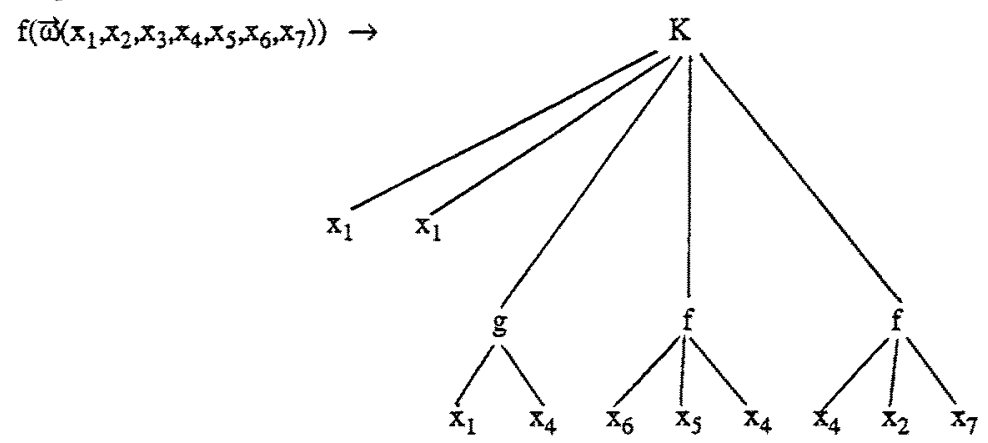

\section{Definition 3.3}

A set of $R$ of rewrite rules is regular if it is of the form $R=U_{f \in D e t} R_{f}$, where $R_{f}$ is a Constrdefinition of $f$, for each $f \in$ Der

For instance, the following systems are regular:

$\left(S_{2}\right)$ Constr $=\{0, s\}$, Der $=\{+$, even $)$ and

$R_{+}: x+0 \rightarrow x, \quad x+s(y) \rightarrow s(x+y)$

$R_{\text {even }}$ : even $(0) \rightarrow$ True, even $(s(0)) \rightarrow$ False, even $(s(s(x))) \rightarrow$ even $(x)$

The Constr-enumeration associated to ' $t$ ' is the one presented hereabove, while the one associated to 'even' is $\omega_{1}=(0), \omega_{2}=(s(0)), \omega_{3}=(s(s(x)))$.

$\left(S_{3}\right)$ Constr $=\{a, \ldots\}$, Der $=\left\{f_{1}(\ldots), f_{2}(\ldots,-)\right\}$ and

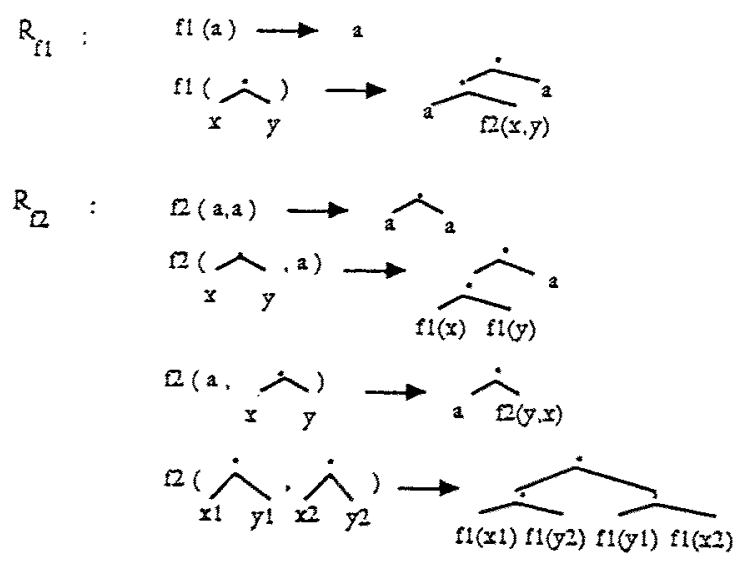

Thus, regular systems can be mutually recursive.

We have the following result :

Theorem 3.1

A regular system is confluent and notherian. Moreover, it provides sufficiently complete and 
hierarchically consistent definitions of the derived operators w.r.t. the constructors.

Proof : confluence is because a regular system has no critical pair. Let us now prove noetherianity. We order $T_{\text {Construder }}$ by the recursive path ordering $>_{\text {rpo }}$ (cf. [Der 82,85]) such that all the constructors are equivalent, all the derived operators are equivalent, and the derived operators are greater than the constructors. Then consicier a rule :

$$
\mathrm{f}\left(\mathrm{w}_{1}\right) \rightarrow \mathrm{K}\left(\mathrm{x}_{1}, \ldots, \mathrm{x}_{\mathrm{m}}, \phi_{1}, \ldots, \phi_{\mathrm{m}}\right)
$$

with the previous notations. Each $\phi_{k}$ is of the form $g\left(y_{1}, \ldots, y_{a r g}\right)$. Since by hypothesis $\vec{\omega}_{i}$ is not empty, the multiset $\left\{\vec{\omega}_{i} \mid\right.$ is strictly greater (for the associated ordering $\gg_{\text {ppo }}$ ) than the multiset $\left(y_{1}, \ldots, y_{\text {ar }(g)}\right\}$. Thus, $f\left(\vec{\omega}_{\mathrm{i}}\right)>_{\text {po }} \phi_{\mathrm{k}}$ for any $\mathrm{k}$, and finally :

$$
f\left(\vec{\omega}_{1}\right)>_{\text {ppo }} \mathrm{K}\left(\mathrm{x}_{1}, \ldots, \mathrm{x}_{\mathrm{n}}, \phi_{1}, \ldots, \phi_{\mathrm{m}}\right)
$$

This ends the proof of the termination of a regular system.

We notice that terms in $T_{\text {Constr }}$ are irreducible, since no left-hand side admits a constructor at the root occurrence. This implies hierarchical consistence w.r.t. the constructors. Conversely, a term in normal form contains no derived operator, since otherwise a rule would apply to further reduce it. Thus, the system is also hierarchically complete.

In this article, we restrict attention to regular systems only. We then have the following result :

Proposition :

Let $R$ be a regular system. For any term $t=f\left(t_{1}, \ldots, t_{n}\right)$ with $f \in$ Der and $\mathfrak{l}_{1}, \ldots, t_{n} \in T_{\text {Constry }}$ the number of rewrite steps between $t$ and its normal from is independent of the rewriting strategy.

Proof : Let us denote by $\Gamma$ the set of terms of $T_{\text {Construder }}$ such that at most one derived operator appears from any path inside the term to the root. It is clear that, if $R$ is regular, if $t \in \Gamma$ and $t \rightarrow{ }^{*} t^{\prime}$, then $t^{\prime} \in \Gamma$. We are going to show, more generally, that for any $t \in \Gamma$, the cost of $t$ does not depend of the evaluation strategy (which proves the previous lemma). Ad absurdum, suppose that this is not the case for a given $t$. We can write $t=K\left[f_{1}\left(\vec{\chi}_{1}\right), \ldots, f_{n}\left(\vec{\chi}_{n}\right)\right]$, where $K$ and the $\vec{\chi}_{i}$ 's are made of constructors only, and the d's are derived operators. We consider two cases :

Case 1 : if $K$ is empty (and $t=f_{1}\left(\overrightarrow{\chi_{1}}\right)$ ), then exactly one rule applies to $t$. We let $\phi(t)$ be the term such that $t \rightarrow \phi(t)$. Then, necessarily, the cost of $\phi(t)$ depends on the evaluation strategy.

Case 2 : else, if the cost of each $\mathrm{f}_{\mathrm{i}}\left(\overrightarrow{\chi_{\mathrm{i}}}\right)$ is equal to $\mathrm{m}_{\mathrm{i}}$, whatever the evaluation strategy, then the cost of $t$ would be $m_{1}+\ldots+m_{n}$, whatever the strategy, which would contradict the hypothesis. Thus, there exists an $i$ such that the cost of $f_{i}\left(\overrightarrow{\chi_{i}}\right)$ depends on the evaluation strategy. We then let $\phi(t)=f_{i}\left(\vec{\chi}_{i}\right)$.

We define the ordering ' $>$ ' by $t>t$ ' iff either $t \rightarrow t^{\prime}$ or $t^{\prime}$ is a strict subterm of $t$. ' $>$ ' is well-founded. Now, the infinite chain :

$$
\mathrm{t}, \phi(\mathrm{t}), \phi(\phi(\mathrm{t})), \ldots
$$

is decreasing for ' $>$ ', which yields the desired contradiction. This terminates the proof.

Note : the property is not true for elements outside of $\Gamma$. Consider for instance the regular system :

$$
x|0 \rightarrow 0, \quad x| s(y) \rightarrow s(x \mid y)
$$


The term ' $(0 \mid 0) 10^{\prime}$ (which is not in $\Gamma$ ) would be nomalized in respectively 1 or 2 steps by an "outermost" or an "innermost" strategy.

We now provide results about the complexity of the rewriting of the derived operators.

\section{Definition 3.4}

Let $f \in$ Der of arity $m$. Let $C_{n}^{f}$ stand for sum the number of rewrite steps of the term $f\left(t_{1}, \ldots, t_{m}\right)$ to its normal form, where the $\left(t_{i}\right)_{1 \leq i \leq m}$ range over $T_{\text {Constr }}$ and are such that $\left|t_{1}\right|+\ldots+\left|t_{m}\right|=n$ :

$$
C_{n}^{f}=\sum_{\substack{t_{1}, \ldots, t_{m} \in T_{\text {Const }} \\\left|t_{1}\right|+\ldots+t_{t_{m}} \mid=n}} \operatorname{cost}\left(f\left(t_{1}, \ldots, t_{m}\right)\right)
$$

The cost series associated to $f$ is :

$$
C^{f}(z)=\sum_{n \geq 0} C_{n}^{f} z^{n}=\sum_{t_{1}, \ldots, t_{m} \in T_{\text {Conut }}} \operatorname{cost}\left(f\left(t_{1}, \ldots, t_{m}\right)\right) z^{\left|t_{1}\right|+\ldots+\left|t_{m}\right|}
$$

From now on, we suppose that Der $=\left\{f_{1}, \ldots, f_{\text {NDer }}\right\}$ where NDer is the number of derived operators. Given a regular system, with each $\mathrm{f}_{\mathrm{i}}$ defined by a Constr-definition, we can write :

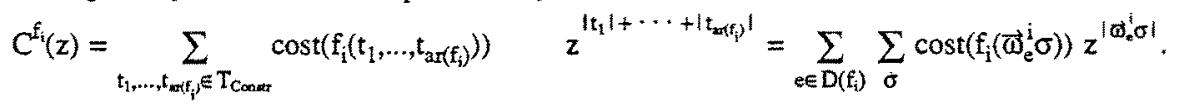

We have : $\operatorname{cost}\left(\mathrm{f}_{\mathrm{i}}\left(\vec{\omega}_{\mathrm{e}}^{\mathrm{i}} \sigma\right)\right)=1+\sum_{1 \leq k \leq \mathrm{n}_{\mathrm{o}}^{\mathrm{i}}} \operatorname{cost}\left(\phi_{\mathrm{k}, \mathrm{i}, \mathrm{e}} \sigma\right)$, in accordance with definition 3.2. Thus :

$$
\begin{aligned}
C^{f_{1}}(z) & =\sum_{e \in D\left(f_{i}\right)} \sum_{\sigma} z^{\left|\omega_{e}^{i} \sigma\right|}+\sum_{e \in D\left(\mathfrak{f}_{1}\right)} \sum_{\sigma} \sum_{1 \leq k<n_{e}} \operatorname{cost}\left(\phi_{k, i, e} \sigma\right) z^{\left|\omega_{e}^{i} \sigma\right|} .
\end{aligned}
$$

Where $A=N^{a r f\left(f_{i}\right)}(z)$ is a constant part of this sum, and $B$ is a recursive part.

In order to simplify the $B$ part of $C^{f_{i}}$, we first notice that it is actually quantified over e corresponding to the rules with non-constant right handsides, that we denote $D_{\mathrm{ac}}\left(f_{\mathrm{i}}\right)$. Let $\mathrm{X}_{\mathrm{e}, \mathrm{i}}$ stand for the variables of $\vec{\omega}_{\mathrm{e}}^{\mathrm{i}}$. Then, we may write $\phi_{\mathrm{k}, \mathrm{i}, \mathrm{e}}=\mathrm{f}_{\mathrm{j}}\left(\mathrm{y}_{1}, \ldots, \mathrm{y}_{\mathrm{ar}\left(\mathrm{f}_{\mathrm{j}}\right)}\right)$, for a certain $\mathrm{j}$, and

$$
\mathrm{X}_{e, \mathrm{i}}-\left\{\mathrm{y}_{1}, \ldots, \mathrm{y}_{\operatorname{ar}\left(\mathrm{f}_{\mathrm{j}}\right)}\right\}=\left\{\mathrm{w}_{1}, \ldots, \mathrm{w}_{1(\mathrm{i}, \mathrm{j}, \mathrm{e})}\right\} \text {, with } 1(\mathrm{i}, \mathrm{j}, \mathrm{e})=\mathrm{X}_{\mathrm{e}, \mathrm{i}} \mathrm{i}-\operatorname{ar}\left(\mathrm{f}_{\mathrm{j}}\right)
$$

where the $w_{i}$ 's are the variables that appear in the left handsides and not in the right handsides of the rules.

Let $\xi_{\mathrm{e}, \mathrm{i}}$ stand for the number of constructors appearing in $\vec{\omega}_{\mathrm{e}}^{\mathrm{l}}$. We suppose that $\sigma$ restricted to the variables $X_{e, i}$ of $\vec{\omega}_{e}^{i}$ is $:\left\{y_{1}:=t_{1}, \ldots, y_{a r\left(f_{j}\right)}:=t_{a r\left(f_{j}\right)}, w_{1}:=t_{1}^{\prime}, \ldots, w_{1(i, j, e)}:=t_{1(i, j, e)}^{\prime}\right\}$.

We have $:\left|\vec{\omega}_{e}^{i} \sigma\right|=\xi_{e, i}+\left|t_{1}\right|+\ldots+\left|t_{a r\left(f_{j}\right)}\right|+\left|t_{1}^{\prime}\right|+\ldots+\left|t_{1(i, j, e)}^{\prime}\right|$

Then :

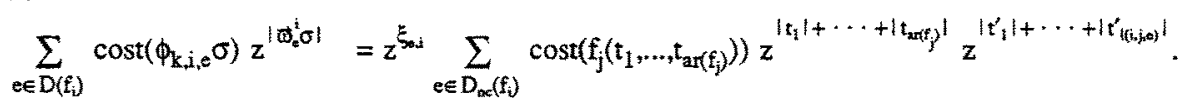

Let $\varepsilon_{e, j}^{i}$ stand for the number of occurrences of ' $f_{j}$ ' in the right handside of the rule $r_{e}^{i}$. The $B$ part of $C^{f_{i}}$ finally rewrites into :

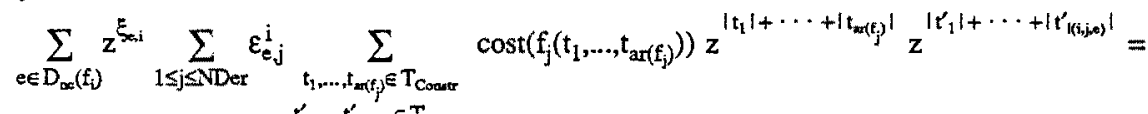

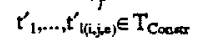

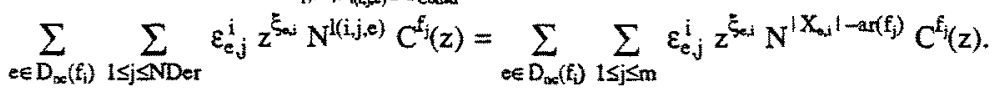




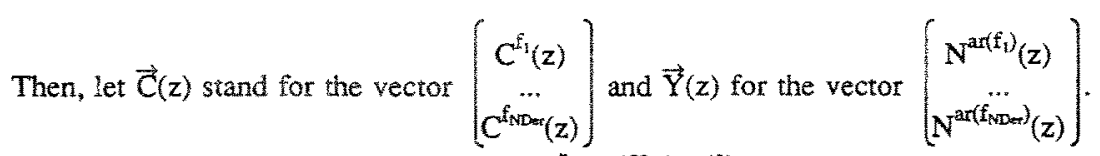

We also define $M_{i, j}(z)=\sum_{e \in D_{n c}\left(f_{i}\right)} \varepsilon_{e, j}^{i} z^{\xi_{0, i}} N^{\left|X_{0, j}\right|-\operatorname{ar}\left(f_{j}\right)}(z)$, and we let $M(z)$ denote the matrix $\left(\mathrm{M}_{\mathrm{i}, j}(\mathrm{z})\right)_{1 \leq \mathrm{i} j \leq \mathrm{NDer}}$ We obtain the central result of this paper:

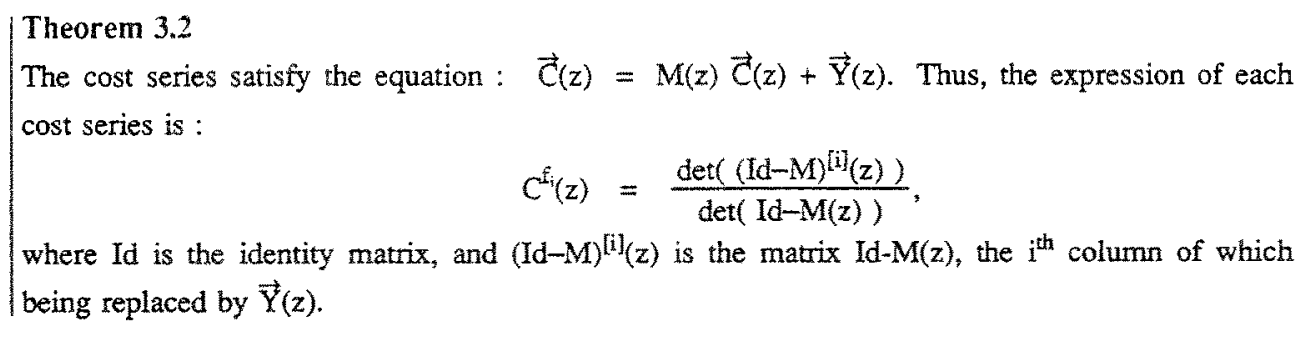

Since $z=\frac{N(z)}{\Phi(N(z))}$, each $C^{f_{i}}(z)$ may be rewritten into the following form :

$$
C^{f_{i}}(z)=\frac{P^{i}(N(z))}{Q^{i}(N(z))}
$$

where $P$ and $Q$ are respectively prime polynomials with integer coefficients. Now, in order to evaluate the $C_{n}^{f_{i}}$, we have to determine the smallest singularity of each $C^{f_{i}}(z)$. Its singularities are :

- either the singularities of $N(z)$, the smallest being for $z=p$,

- or the $z^{\prime}$ s such that $Q^{i}(N(z))=0$. Let us denote by $\rho_{0}^{i}$ the smallest real positive root of $Q^{i}\left(N\left(\rho_{0}^{i}\right)\right)=0$ (with the convention that $\rho_{0}^{i}=\infty$ if the equation $Q^{i}(N(z))$ has no root for $\left.|z| \leq p_{0}^{i}\right)$.

We now have the following main theorem:

$$
\begin{array}{ll}
\text { Theorem 3.3 } & \\
\text { (3) if } \rho<\rho_{0} \text {, then: } & \bar{C}_{n}^{f_{i}}=k_{1}\left(1+O\left(\frac{1}{n}\right)\right) \\
\text { (2) if } \rho=\rho_{0}^{i} \text {, then: } & \bar{C}_{n}^{f_{i}}=k_{2} n^{m_{2} / 2}\left(1+O\left(\frac{1}{\sqrt{n}}\right)\right) \\
\text { (3) if } \rho>\rho_{0}^{i} \text {, then: } & \bar{C}_{n}^{f_{i}}=k_{3}\left(\frac{\rho}{\rho_{0}^{i}}\right)^{n} n^{m_{3}+1 / 2}\left(1+O\left(\frac{1}{\sqrt{n}}\right)\right)
\end{array}
$$

The $k_{j}$ 's are real numbers, and the $m_{j}$ 's are strictly positive integers; all of them can be expressed simply (as shown hereafter - Results 3.4,3.5 and 3.6).

We now proceed by proving theorem 3.3 by considering successively the three cases.

\section{Study of case (1)}

We can write, using Taylor-Izritch expansion formula :

$$
\frac{P_{i}(N(z))}{Q_{i}(N(z))}=\frac{P_{i}(\tau)}{Q_{i}(\tau)}+\frac{\partial}{\partial N}\left[\frac{P_{i}}{Q_{i}}\right]_{1 N=\tau}(N(z)-t)+\frac{\partial^{2}}{\partial N^{2}}\left[\frac{P_{i}}{Q_{i}}\right]_{\mid N=i}(N(z)-\tau)^{2}+O\left(|N(z)-\tau|^{2}\right)
$$

Applying the transfer lemmas of section 2, and using the approximation : 


$$
N(z)=\tau-\sqrt{\frac{2 \Phi(\tau)}{\Phi^{\prime \prime}(\tau)}}\left[1-\frac{z}{\rho}\right]^{1 / 2}+\gamma\left(1-\frac{z}{\rho}\right)+0\left[\left(1-\frac{z}{\rho}\right]^{3 / 2}\right]
$$

we get :

$$
C_{n}^{f}=k_{1} \sqrt{\frac{\Phi(\tau)}{2 \pi \Phi^{\prime \prime}(\tau)}} \rho^{-n_{n} n^{-3 / 2}}\left(1+O\left(\frac{1}{n}\right)\right), \text { with } k_{1}=\frac{1}{\operatorname{ar}\left(f_{i}\right) \tau^{\operatorname{arf}\left(f_{i}\right)-1}} \frac{\partial}{\partial N}\left[\frac{P_{i}}{Q_{i}}\right]_{1 N=\tau},
$$

and finally :

Result 3.4

$$
\bar{C}_{n}^{\mathrm{f}}=k_{1}\left(1+O\left(\frac{1}{n}\right)\right) \text {, with } k_{1}=\frac{1}{\operatorname{ar}\left(f_{i}\right) \tau^{\operatorname{ar}\left(f_{i}\right)-1}} \frac{\partial}{\partial N}\left[\frac{P_{i}}{Q_{i}}\right]_{\mid N=\tau}
$$

\section{Example 3.1}

We consider, on binary trees built as previously, another version of a shuffle function on trees, defined by the following set of rules :

$$
\begin{aligned}
& R_{i l} \quad T(a, a) \longrightarrow a
\end{aligned}
$$

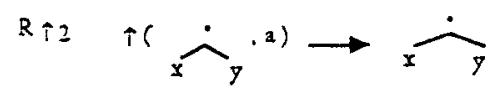

$$
\begin{aligned}
& R_{\uparrow 3}, \quad T(2, \underbrace{\prime}_{y}) \rightarrow g(x, y)
\end{aligned}
$$

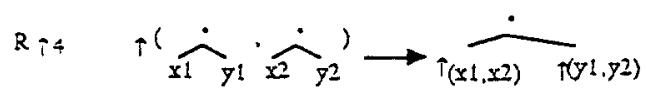

$$
\begin{aligned}
& R_{g 1} \quad g(a, a) \longrightarrow \underset{a}{\dot{\gamma}_{a}} \\
& R_{g} 2 \quad g\left({\underset{x}{y}}^{2}\right) \rightarrow g(x, y) \\
& \mathrm{Rg}^{3} \quad \mathrm{~g}(\mathrm{a}, \underset{x}{\dot{y}}) \rightarrow g(x, y)
\end{aligned}
$$

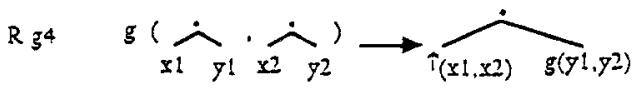

The matrix $M(z)$ associated to the $\uparrow$ and $g$ is :

$\left[\begin{array}{cc}2 z^{2} N^{2}(z) & z^{2} \\ z^{2} N^{2}(z) & 2 z^{2}+z^{2} N^{2}(z)\end{array}\right]$, and $Y(z)=\left[\begin{array}{l}N^{2}(z) \\ N^{2}(z)\end{array}\right]$. This yields, after computation of the determinants, and replacement of $z$ by $\frac{N(z)}{1+N^{2}(z)}$ (these computations have been performed with assistance of the 
MAPLE program [MAPLE 85]) :

$$
\begin{aligned}
& C^{\dagger}(z)=\frac{\left(1+N^{2}(z)+N^{4}(z)\right) N^{2}(z)\left(1+N^{2}(z)\right)^{2}}{1+2 N^{2}(z)-N^{4}(z)-N^{6}(z)}=\frac{P_{1}(N(z))}{Q_{1}(N(z))} \\
& C^{g}(z)=\frac{\left(1+2 N^{2}(z)+N^{6}(z)\right) N^{2}(z)\left(1+N^{2}(z)\right)^{2}}{1+2 N^{2}(z)-N^{4}(z)-N^{6}(z)}=\frac{P_{2}(N(z))}{Q_{2}(N(z))}
\end{aligned}
$$

The denominator $\left(1+2 N^{2}-N^{4}-N^{6}\right)$ has no root for $N \in[0,1]$. We are therefore in the current case (1). Computarion gives: $\frac{\partial}{\partial N}\left(\frac{P_{1}}{Q_{1}}\right]_{1 N=1}=144$ and $\frac{\partial}{\partial N}\left[\frac{P_{2}}{Q_{2}}\right]_{1 N=1}=200$. Finally :

$$
\begin{aligned}
& \bar{C}_{n}^{\uparrow}=72\left(1+O\left(\frac{1}{n}\right)\right) \\
& \bar{C}_{n}^{g}=100\left(1+O\left(\frac{1}{n}\right)\right) .
\end{aligned}
$$

End of example 3.1

Study of case (2)

We can write :

$$
\frac{P_{i}(N(z))}{Q_{i}(N(z))}=\frac{1}{(N(z)-\tau)^{s}} \frac{P_{i}(N(z))}{\bar{Q}_{i}(N(z))},
$$

where $s$ is a strictly positive integer, and $\bar{Q}_{i}$ is an integer polynomial such that $\bar{Q}_{i}(\tau) \neq 0$. Then :

$$
\frac{P_{i}(N(z))}{Q_{i}(N(z))}=\frac{P_{i}(\tau)}{\bar{Q}_{i}(\tau)}(N(z)-\tau)^{-s}+\gamma_{2}(N(z)-\tau)^{-s+1 / 2}+O\left(|N(z)-\tau|^{-s+1}\right)
$$

Applying the transfer lemmas of section 2, and using the approximation (F1), we obtain :

$$
\mathrm{C}_{\mathrm{n}}^{\mathrm{f}}=\mathrm{k}_{2} \rho^{-\mathrm{n}^{-\mathrm{n}^{2}}}{ }^{\frac{s}{2}}\left(1+\mathrm{O}\left(\frac{1}{\mathrm{n}^{1 / 2}}\right)\right), \text { with } \mathrm{k}_{2}^{\prime}=(-1)^{s} \frac{\mathrm{P}_{\mathrm{i}}(\tau)}{\overline{\mathrm{Q}}_{\mathrm{i}}(\tau)}\left[\frac{2 \Phi(\tau)}{\Phi^{\prime \prime}(\tau)}\right]^{-\frac{s}{2}} \frac{1}{\Gamma(\mathrm{s} / 2)},
$$

and thus :

$$
\bar{C}_{n}=k_{2} n^{\frac{s+1}{2}}\left(1+O\left(\frac{1}{n^{1 / 2}}\right)\right) \text {, with } k_{2}=(-1)^{s-1} \frac{1}{\operatorname{ar}\left(f_{i}\right) \tau^{\operatorname{ar}\left(f_{i}\right)-1}} \frac{P_{i}(\tau)}{\bar{Q}_{i}(\tau)}\left[\frac{2 \Phi(\tau)}{\Phi^{\prime \prime}(\tau)}\right)^{\frac{(s+1)}{2}} \frac{\Gamma(-1 / 2)}{\Gamma(s / 2)},
$$

Finally, using $\Gamma(1 / 2)=\sqrt{\pi}$ and $\Gamma(s / 2)=\left\{\begin{array}{ll}(p-1) ! & \text { if } s=2 p \\ \frac{(2 p-1) !}{(p-1) !} \frac{1}{2^{2 p+1}} & \text { if } s=2 p+1\end{array}\right.$, we obtain :

\section{Result 3,5}

$\overline{\mathrm{C}}_{\mathrm{n}}^{\mathrm{f}_{\mathrm{i}}}=\mathrm{k}_{2^{\mathrm{n}}}{ }^{\frac{s+1}{2}}\left(1+\mathrm{O}\left(\frac{1}{\mathrm{n}^{1 / 2}}\right)\right)$,

with

$$
\begin{aligned}
& k_{2}=-\frac{1}{\operatorname{ar}\left(f_{i}\right) \tau^{\operatorname{ar}\left(f_{i}\right)-1}} \frac{P_{i}(\tau)}{\bar{Q}_{i}(\tau)} \quad\left[\frac{2 \Phi^{\prime \prime}(\tau)}{\Phi(\tau)}\right]^{-(p+1)} \frac{\sqrt{\pi}(p-1) !}{(2 p-1) ! 4^{p-1}} \quad \text { if } s=2 p \\
& s_{2}=\frac{1}{\operatorname{ar}\left(f_{i}\right) \tau^{\operatorname{ar}\left(f_{i}\right)-1}} \frac{P_{1}(\tau)}{\bar{Q}_{i}(\tau)} \quad\left[\frac{2 \Phi^{\prime \prime}(\tau)}{\Phi(\tau)}\right]^{-\frac{2 p+1}{2}} \frac{2 \sqrt{\pi}}{(p-1) !} \quad \text { if } s=2 p+1
\end{aligned}
$$




\section{Example 3.2}

We consider the same system as in example 3.1 , except that rule $R_{\uparrow 2}$ is replaced by rule $R_{\uparrow z^{\prime}}$ :

$$
R \uparrow 2^{\prime} \uparrow(\overbrace{x}^{\prime}, a) \longrightarrow g(x, y)
$$

The matrix $M(z)$ associated to the $\uparrow$ and $g$ is now :

We obtain, after replacement of $z$ by $\frac{N(z)}{1+N^{2}(z)}$ :

$$
\left[\begin{array}{cc}
2 z^{2} N^{2}(z) & 2 z^{2} \\
z^{2} N^{2}(z) & 2 z^{2}+z^{2} N^{2}(z)
\end{array}\right]
$$

$$
\operatorname{det}(I-M(z))=(1-N(z)) \frac{\left(N^{2}(z)+2\right)(N(z)+1)}{\left(1+N^{2}(z)\right)^{3}}
$$

which yields :

$$
\begin{aligned}
& C^{\uparrow}(z)=\frac{1}{1-N(z)} \frac{N^{2}(z)\left(1+N^{2}(z)\right)}{1+N(z)}=\frac{1}{1-N(z)} \frac{P_{1}(N(z))}{\bar{Q}_{1}(N(z))} \\
& C^{g}(z)=\frac{1}{1-N(z)} \frac{N^{2}(z)\left(1+N^{2}(z)\right)}{1+N(z)}=\frac{1}{1-N(z)} \frac{P_{2}(N(z))}{\bar{Q}_{2}(N(z))}
\end{aligned}
$$

We are in the current case (1). Computation gives: $\frac{P_{1}(1)}{\bar{Q}_{1}(1)}=\frac{P_{2}(1)}{\bar{Q}_{2}(1)}=1$, and finally :

$$
\begin{aligned}
& \overline{\mathrm{C}}_{\mathrm{n}}^{\uparrow}=\sqrt{\frac{\pi}{2}} \mathrm{n}^{1 / 2}\left(1+\mathrm{O}\left(\frac{1}{\sqrt{\mathrm{n}}}\right)\right) \\
& \overline{\mathrm{C}}_{\mathrm{n}}^{\mathrm{g}}=\sqrt{\frac{\pi}{2}} \mathrm{n}^{1 / 2}\left(1+\mathrm{O}\left(\frac{1}{\sqrt{\mathrm{n}}}\right)\right)
\end{aligned}
$$

End of example 3.2

\section{Study of case (3)}

Let $\tau_{0}^{\mathrm{i}}=\mathrm{N}\left(\rho_{0}^{\mathrm{i}}\right)$. We have $: 0<\rho_{0}^{\mathrm{i}}<\rho$, and $0<\tau_{0}^{\mathrm{i}}<\tau$. We can write :

$$
\frac{P_{i}(N(z))}{Q_{i}(N(z))}=\frac{1}{\left(N(z)-\tau_{0}^{i}\right)^{s}} \frac{P_{i}(N(z))}{\bar{Q}_{i}(N(z))}
$$

where $s$ is a strictly positive integer, and $\bar{Q}_{i}$ is an integer polynomial such that $\bar{Q}_{j}\left(\tau_{0}^{i}\right) \neq 0$. Then, using a Taylor-Izritch expansion of $z=\frac{N(z)}{\Phi(N(z))}$ in the neighbourhood of $\rho_{0}^{i}$, we get :

$$
z=\frac{N(z)}{\Phi(N(z))}=\frac{\tau_{0}^{i}}{\Phi\left(\tau_{0}^{i}\right)}+\frac{\partial}{\partial N}\left[\frac{N}{\Phi(N)}\right]_{\mid N=N_{0}}\left(N-\tau_{0}^{i}\right)+O\left(\left|z-\rho_{0}^{i}\right|^{2}\right),
$$

from which we derive :

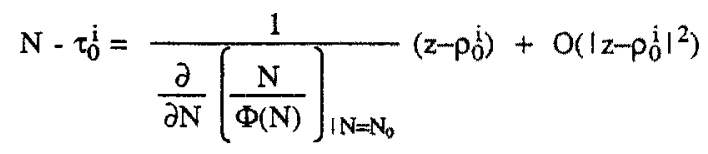




$$
=\frac{-1}{\frac{1}{\tau_{0}^{i}}-\frac{\Phi^{\prime}\left(\tau_{0}^{i}\right)}{\Phi\left(\tau_{0}^{i}\right)}}\left(1-\frac{z}{\rho_{0}^{i}}\right)+O\left(11-\frac{z}{\rho_{0}^{i}} l^{2}\right) .
$$

Using this developement in the previous expression of $\frac{\mathrm{P}_{\mathrm{i}}(\mathrm{N}(\mathrm{z}))}{\mathrm{Q}_{\mathrm{j}}(\mathrm{N}(\mathrm{z}))}$ yields :

$$
\frac{P_{i}(N(z))}{Q_{i}(N(z))}=(-1)^{s}\left(1-\frac{z}{\rho_{0}^{i}}\right)^{-s}\left[\frac{1}{\tau_{0}^{i}}-\frac{\Phi^{\prime}\left(\tau_{0}^{i}\right)}{\Phi\left(\tau_{0}^{i}\right)}\right]^{s} \frac{P_{i}\left(\tau_{0}^{i}\right)}{\bar{Q}_{i}\left(\tau_{0}^{i}\right)}+0\left[\left|1-\frac{z}{\rho_{0}^{i}}\right|^{-s+\frac{1}{2}}\right]
$$

Thus,

$$
C_{n}^{f}=(-1)^{s}\left[\frac{1}{\tau_{0}^{i}}-\frac{\Phi^{\prime}\left(\tau_{0}^{i}\right)}{\Phi\left(\tau_{0}^{i}\right)}\right]^{s} \frac{P_{i}\left(\tau_{0}^{i}\right)}{\bar{Q}_{i}\left(\tau_{0}^{i j}\right)}\left(\rho_{0}^{i}\right)^{-n} \frac{n^{s-1}}{\Gamma(s-1)}\left(1+O\left(\frac{1}{\sqrt{n}}\right)\right)
$$

and finally :

\section{Result $\mathbf{3 . 6}$}

$$
\begin{aligned}
& \overline{\mathrm{C}}_{\mathrm{n}}^{\mathrm{f}}=\mathrm{k}_{3}\left(\frac{\rho}{\rho_{0}^{i}}\right)^{\mathrm{n}} \mathrm{n}+\frac{1}{2}\left(1+O\left(\frac{1}{\sqrt{n}}\right)\right) \\
& \text { with } k_{3}=\frac{(-1)^{s}}{(s-1) !} \frac{1}{\operatorname{ar}\left(f_{i}\right) \tau^{a r\left(f_{i}\right)-1}}\left[\frac{1}{\tau_{0}^{i}}-\frac{\Phi^{\prime}\left(\tau_{0}^{i}\right)}{\Phi\left(\tau_{0}^{i}\right)}\right)^{s} \frac{P_{i}\left(\tau_{0}^{i}\right)}{\bar{Q}_{i}\left(\tau_{0}^{i}\right)} \sqrt{\frac{2 \pi \Phi^{\prime \prime}\left(\tau_{0}^{1}\right)}{\Phi\left(\tau_{0}^{i}\right)}}
\end{aligned}
$$

\section{Example 3.3}

We consider the same system as in example 3.1 (or example 3.2 ), except that rule $R_{\uparrow_{2}}$ (or rule $R_{\uparrow 2}$ ) is replaced by rule $R_{\uparrow_{2}}$ :

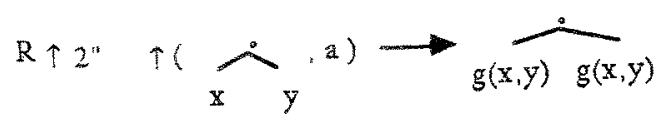

The matrix $\mathrm{M}(\mathrm{z})$ associated to the $\uparrow$ and $\mathrm{g}$ is now :

$$
\left[\begin{array}{cc}
2 z^{2} N^{2}(z) & 3 z^{2} \\
z^{2} N^{2}(z) & 2 z^{2}+z^{2} N^{2}(z)
\end{array}\right]
$$

This yields, after computation of the determinants, and replacement of $z$ by $\frac{N(z)}{1+N^{2}(z)}$ :

$$
\begin{aligned}
& C^{\uparrow}(z)=\frac{\left(1+N^{2}(z)\right)^{2}\left(1+3 N^{2}(z)\right) N^{2}(z)}{1+2 N^{2}(z)-N^{4}(z)-3 N^{6}(z)} \\
& C^{g}(z)=\frac{\left(1+N^{2}(z)\right)^{2}\left(1+2 N^{2}(z)\right) N^{2}(z)}{1+2 N^{2}(z)-N^{4}(z)-3 N^{6}(z)}
\end{aligned}
$$

The expression $\left(1+2 N^{2}(z)-N^{4}(z)-3 N^{6}(z)\right)$ admits a root for $N_{0} \sim 0.93336$, which gives $\tau_{0} \sim$ $0.49881(<\tau=1 / 2)$. We are therefore in the current case (3), and computation gives finally :

$$
\bar{C}_{n}^{\hat{\imath}}=k_{3}\left[\frac{\rho}{\rho_{0}^{i}}\right]^{n} \sqrt{n}\left(1+O\left(\frac{1}{\sqrt{n}}\right)\right)
$$




$$
\begin{aligned}
& \overline{\mathrm{C}}_{\mathrm{n}}^{\mathrm{g}}=\mathrm{k}_{3}^{\prime}\left(\frac{\rho}{\rho_{0}^{i}}\right)^{\mathrm{a}} \sqrt{\mathrm{n}}\left(1+O\left(\frac{1}{\sqrt{n}}\right)\right) \\
& \text { with } \mathrm{k}_{3} \sim 0.27901, \mathrm{k}_{3}^{\prime} \sim 0.21234, \text { and } \frac{\rho}{\rho_{0}^{i}} \sim 1.00238 \\
& \text { End of example } 3.3
\end{aligned}
$$

We notice that, simply modifying one rule between examples $3.1,3.2$ and 3.3 , induces respectively constant, polynomial or exponential cost. This illustrates the great sensitivity of the cost of rewriting w.r.t. mild modifications within the rewrite rules.

Thus, in each particular case, the asymptotic developments are obtained with very few computations, that just rely on the "geometry" of the system. The user of our methods actually never needs to manipulate formal series; (s)he simply has to apply theorems 3.3, 3.4 or 3.6 (according to the singularity closest to the origin of the $\mathrm{Q}_{\mathrm{i}}(\mathrm{N}(\mathrm{z}))$ 's.

\section{Conclusion}

For the class of the regular term rewriting systems, we have provided ways of obtaining asymptotic evaluations of the cost series. The user does not need to actually manipulate formal series, since our results are given under the form of ready-to-use formulae. These results solely depend on physical characteristics of the system, easily obtainable : number of variables and of constructors in the lefthand sides, occurrences of derived operators in the right-hand sides. Then, the average cost is constant, polynomial or exponential, according to the position of the singularity of the expressions $Q_{i}(N(z))$ closest to the origin.

\section{Acknowledgements}

We thank Jean-Pierre Jouannaud for contribution to the proof of Theorem 3.1 and Philippe Flajolet for fruitfull discussions. This work has been partially supported by the C.N.R.S. P.R.C. de Programmation, and the ESPRIT Meteor project.

\section{References}

[Bel 85] Belhassen S., "Séries formelles de complexité dans les types abstraits algébriques", Rapport de DEA, Orsay 1985.

[BBWT 81] Bergstra J.A., Broy M., Wirsing M., Tucker J.V., "On the power of algebraic specifications", Proc. of the M.F.C.S. Conference, L.N.C.S. 118, Springer Verlag, 1981.

[BCV 85] Bidoit M., Choppy C., Voisin F., "The Asspegique specification environment : motivations and design", Proc. 3rd Workshop on Theory and Applications of Abstract data types", Bremen, Nov. 
1984. Recent Trends on Data Type Specification (H.-J. Kreowski ed.), Informatik Fachberichte 116, Springer-Verlag, Berlin-Heidelberg, 1985.

[C\&K 83] Choppy C., Kaplan S., "Complexity calculus for abstract data types", LRI Report no 147, Nov. 1983.

[CLR 80] Choppy C., Lescanne P., Rémy J.-L., "Improving abstract data type specification by appropriate choice of constructors", Proc. Intern. Workshop on Program Construction, Bonas, France, 1980, Mac Millan, A. Biemann, G. Guiho and Y. Kodratoff (eds), 1983.

[Der 82] N. Dershowitz, "Orderings for term-rewriting systems", T.C.S. vol. 17.3, March 1982.

[Der 85] N. Dershowitz, "Termination", Proc. of the RTA'85 Conference, L.N.C.S. 202, 1985.

[E\&M 81] Ehrig H., Mahr B., "Complexity of algebraic implementations for abstract data types", J. of Computer and System Sciences, vol 23, no 2, Oct, 1981, pp. 223-253.

[Fla 87] Flajolet P., "The symbolic operator method", in Mathematical methods in the analysis of algorithms and data structures, L.N.C.S., Springer-Verlag, to appear 1987.

[F\&S 82] Flajolet P., Steyaert I.M., "A complexity calculus for classes of recursive search programs over tree structures", Proc. 22nd IEEE Symp. on Foundations of Computer Science, Nashville, 1982, pp. 386,-393.

[F\&G 84] Forgaard R., Gutag J.V., "REVE : a term rewriting system generator with failure-resistant Knuth-Bendix", Proc. of an NSF Workshop on the rewrite rule laboratory, Report no 84GEN008, General Electric, Apr. 1984.

[FGJM 84] Futatsugi K., Goguen J.A., Jouannaud J.P., Meseguer J., "Principles of OBJ2", CRIN Report 84-R-066.

[GSF 86] Gaudel M.C., Soria M., Froidevaux C., "Types de données et algorithmes - vol 1 : Analyse d'algorithmes, Définition des types de données", Collection Didactique, INRIA, 1986.

[GHW 85] Guttag J.V., Horning J.J., Wing J.M., "Larch in five easy pieces" Digital System Research Center Report, Jul. 1985.

[Kap 86] Kaplan S., "A compiler for conditional tern rewriting systems" Proc. of the RTA'87 Conference, L.N.C.S. this volume, also : L.R.I. Report no 315, Dec. 1986.

[Knu 68] Knuth D., "The ant of computer programming : Fundamental algorithms", Addison Wesley, Reading, 1968.

[Knu 73] Knuth D., "The ant of computer programming : Sorting and searching", Addison Wesley, Reading, 1973. 
[M\&M 78] Meir A., Moon J.W., "On the altitude of nodes in random trees", Canadian Joumal of Math. 30, 1978, pp. 997-1015.

[MAPLE 85] Char B.W., Geddes K.O., Gonnet G.H., Watt S.M., "MAPLE : Reference Manual", University of Waterloo, 1985.

[S\&S 84] Soria M., Steyaert J.M., "Average efficiency of pattern matching on Lisp expressions", Proc. of the CAAP 84 Conference, also : LRI Report no 178, May 1984.

[Stey 84] Steyeart J.M., "Complexité et structure des algorithmes", Thèse d'Etat, Université de Paris 7, 1984. 